\title{
Classificatore genetico predittivo della benignità di noduli tiroidei a citologia indeterminata
}

\author{
Cristina Romei ${ }^{1} \cdot$ Rossella Elisei $^{1}$
}

Accettato: 7 aprile 2020 / Pubblicato online: 1 settembre 2020

(c) The Author(s) 2020

Commento a:

A thyroid genetic classifier correctly predicts benign nodules with indeterminate cytology: two independent, multicentric, prospective validation trials.

M. Zafereo, B. McIver, S. Vargas-Salas,

J.M. Domínguez, D.L. Steward, F.C. Holsinger,

E. Kandil, M. Williams, F. Cruz, S. Loyola, A. Solar,

J.C. Roa, A. León, N. Droppelman, M. Lobos, T. Arias,

C.S. Kong, N. Busaidy, E.G. Grubbs, P. Graham,

J. Stewart, A. Tang, J. Wang, L. Orloff, M. Henríquez,

M. Lagos, M. Osorio, D. Schachter, C. Franco,

F. Medina, N. Wohllk, R.E. Diaz, J. Veliz, E. Horvath,

H. Tala, P. Pineda, P. Arroyo, F. Vasquez, E. Traipe,

L. Marín, G. Miranda, E. Bruce, M. Bracamonte,

N. Mena, H.E. González.

Thyroid (2020) 30(5):704-712

Nonostante l'analisi citologica sia ancora il gold standard per la diagnosi del nodulo tiroideo, in alcuni casi la risposta della citologia non è in grado di determinare se un nodulo è maligno o benigno e, in questi casi, l'analisi istologica del pezzo operatorio risulta ancora l'unica possibilità diagnostica. Negli ultimi anni sono stati messi a punto test molecolari per meglio chiarire l'esatta natura del nodulo a citologia indeterminata, in modo tale da evitare interventi di tiroidectomia assolutamente non necessari. Nel corso degli anni c'è stata un'evoluzione delle tecniche molecolari di analisi che hanno poi portato allo sviluppo di alcuni pannelli di analisi multigenica (ThyroSeq, Afirma) [2, 3] in grado di identificare con alta specificità e sensibilità la natura maligna $o$

\section{R. Elisei}

relisei@endoc.med.unipi.it; rossella.elisei@med.unipi.it

1 U.O. Endocrinologia, Dipartimento di Medicina Clinica e Sperimentale, Università di Pisa, Pisa, Italia benigna del nodulo tiroideo. La maggior parte dei test proposti per la caratterizzazione del nodulo tiroideo sono però disponibili solo in laboratori altamente specialistici per cui, in molti ospedali, la chirurgia continua ad essere utilizzata per la diagnosi del nodulo tiroideo a citologia indeterminata.

Gli autori di questo studio avevano precedentemente pubblicato lo sviluppo di un test genetico a 10 geni in grado di identificare i noduli benigni a citologia indeterminata con un valore predittivo negativo del $96 \%$ e una specificità dell' $87 \%$ [1].

Nel presente articolo gli stessi autori riportano i risultati dello studio di validazione del test genetico precedentemente proposto e ne mostrano la performance. A tal fine sono stati eseguiti due trial multicentrici indipendenti e prospettici, condotti su popolazioni etnicamente diverse (TGCT1/Cile e TGCT-2/USA). Sono stati raccolti un totale di 4.061 agoaspirati derivanti da 15 diversi centri: 897 casi risultavano indeterminati all'esame citologico. Lo studio di validazione è stato condotto però solo in 270 casi perché molti pazienti non sono stati operati mentre un altro gruppo è stato escluso per motivi tecnici. l'esame istologico 45/155 (26\%) e 41/115 (39\%) casi erano risultati maligni. Di questi, il test genetico aveva identificato come maligni 37 e 41 casi rispettivamente, con una sensibilità rispettivamente del 90 e $91 \%$. Centoquattordici e 70 casi erano invece risultati benigni e di questi 101 e 61 erano correttamente identificati come benigni anche dal test genetico con una specificità dell' 89 e $87 \%$ nelle due coorti. Il valore predittivo negativo era 96 e $94 \%$, mentre il valore predittivo positivo era 74 e $82 \%$. L'accuratezza generale del test è risultata $89 \%$. Gli autori concludono che il test genetico presentato nel loro studio ha un'ottima performance, che può identificare con un'ottima specificità la natura benigna dei noduli tiroidei e può essere applicato nella pratica clinica per la diagnosi dei noduli tiroidei a 
citologia indeterminata diminuendo, quindi, il numero degli interventi chirurgici non necessari.

Questo studio di validazione risulta essere significativo perché è stato condotto su popolazioni etnicamente diverse ottenendo, però, risultati assolutamente sovrapponibili. Lo studio proposto non è affetto da bias nei criteri di selezione perché i casi erano stati raccolti in rapporto simile sia in centri accademici che in ospedali di comunità, rendendo quindi il campione omogeneo.

La cosa forse più rilevante di questo studio è rappresentata dal fatto che questo test significativamente validato è stato trasformato in un kit diagnostico la cui semplicità tecnica e grande accuratezza potrà esser di notevole aiuto per il clinico nella diagnosi prechirurgica del nodulo tiroideo.

Funding Note Open access funding provided by Università di Pisa within the CRUI-CARE Agreement.

Nota della casa editrice Springer Nature rimane neutrale in riguardo alle rivendicazioni giurisdizionali nelle mappe pubblicate e nelle affiliazioni istituzionali.

Open Access This article is licensed under a Creative Commons Attribution 4.0 International License, which permits use, sharing, adap- tation, distribution and reproduction in any medium or format, as long as you give appropriate credit to the original author(s) and the source, provide a link to the Creative Commons licence, and indicate if changes were made. The images or other third party material in this article are included in the article's Creative Commons licence, unless indicated otherwise in a credit line to the material. If material is not included in the article's Creative Commons licence and your intended use is not permitted by statutory regulation or exceeds the permitted use, you will need to obtain permission directly from the copyright holder. To view a copy of this licence, visit http://creativecommons.org/licenses/by/4.0/.

\section{Bibliografia}

1. Gonzalez HE, Martınez JR, Vargas-Salas S et al (2017) A 10gene classifier for indeterminate thyroidnodules: development and multicenter accuracy study. Thyroid 27:1058-1067

2. Alexander EK, Schorr M, Klopper J et al (2014) Multicenter clinical experience with the Afirma gene expression classifier. J Clin Endocrinol Metab 99:119-125

3. Nikiforova MN, Mercurio S, Wald AL et al (2018) Analytical performance of the Thyroseq V3 genomic classifier for cancer diagnosis in thyroid nodules. Cancer 124:1682-1690 